Mercedes Freire, Silvia Peralta, Efrén Mendoza, Jessica Linzán. 


\title{
Parque temático turístico cultural como alternativa de diversificación de la oferta turística en la ciudad de Santa Elena.
}

\section{Touristic theme park cultural as an alternative of diversification of the tourist offer in the city of Santa Elena}

\author{
Mercedes E. Freire, Silvia P. Peralta, Efrén A. Mendoza, Jessica S. Linzán \\ Facultad de Ciencias Administrativas, Carrera de Gestión y Desarrollo Turístico \\ Universidad Estatal Península de Santa Elena, \\ e-mail: mfreire@upse.edu.ec,
}

\begin{abstract}
Resumen
El turismo de sol y playa es el segmento que genera mayor flujo de turistas y representa un motor importante para el desarrollo de la actividad en muchos países. Sin embargo, el hacinamiento, el deterioro ambiental, los cambios en la demanda y el aumento de la competencia han generado preocupación por el futuro de los destinos consolidados de sol y playa. Dada esta afirmación, y siendo Santa Elena una localidad con estas características, se hace relevante buscar alternativas de diversificación de la oferta turística que permitan dinamizar procesos de desarrollo sostenible del sector. El Objetivo de esta investigación, consiste en determinar el parque temático turístico cultural como alternativa de diversificación de la oferta turística en la ciudad de Santa Elena. La metodología utilizada se basó en un enfoque cualitativo-cualitativo apoyado en la recolección de datos con el diseño de entrevistas en profundidad con actores comunitarios como informantes clave para determinar las categorías a evaluar y establecer elementos esenciales del diagnóstico. Los principales resultados determinaron que en la ciudad de Santa Elena a pesar de la existencia del museo Amantes de Sumpa con enfoque cultural no hay lugar o atracción turística con temática que relacione lo recreativo y cultural, representando costumbres y tradiciones locales para la satisfacción de los visitantes.
\end{abstract}

Palabras Clave: Parque Temático, Desarrollo Local, Diversificación turística.

\begin{abstract}
The tourism of sun and beach is the segment that generates greater flow of tourists and represents an important motor for the development of the activity in many countries. However, overcrowding, environmental deterioration, changes in demand and increased competition have generated concern about the future of consolidated sun and beach destinations. Given this assertion, and being Santa Elena a locality with these characteristics, it becomes relevant to look for alternatives of diversification of the tourist supply that allow to dynamize processes of sustainable development of the sector. The objective of this research is to determine the cultural tourism theme park as an alternative to diversify tourism in the city of Santa Elena. The methodology used was based on a qualitative-qualitative approach based on the collection of data with the design of indepth interviews with community actors as key informants to determine the categories to be evaluated and to establish essential elements of the diagnosis. The main results determined that in the city of Santa Elena despite the existence of the museum Lovers of Sumpa with cultural focus there is no place or tourist attraction with thematic that relates the recreational and cultural, representing customs and local traditions for the satisfaction of the visitors ..
\end{abstract}

Key words: Theme park, Local Development, Tourism diversification. 


\section{Introducción}

A nivel mundial se han producido cambios importantes en la demanda turística, en este contexto, se han ido desplegando nuevas motivaciones en la experiencia del turista. El nuevo visitante busca variadas opciones en los destinos turísticos, más allá del sol y playa y demanda productos más especializados, por lo que, las zonas litorales deben orientarse a procurar nuevas ofertas turísticas y atender a nuevos nichos de mercado que se proponen en el contexto del turismo. La diversificación es una estrategia que está orientada a incorporar nuevos elementos recreativos especialmente en segmentos de demanda actual, que pretendan atender las nuevas perspectivas motivacionales del turista. Todo esto nos conlleva a profundizar el mejoramiento de nuevas estrategias que ayuden enfrentar algunos elementos negativos que se presentan en las zonas litorales, como la estacionalidad y la falta de empleo. Podemos inferir entonces, que la diversificación de la oferta turística nos llevaría a fomentar nuevos negocios a la población y por ende a una mejor calidad de vida que en última instancia también es el objetivo del desarrollo turístico.

El objetivo de esta investigación, es determinar al parque temático turístico cultural como alternativa de diversificación de la oferta turística en la ciudad de Santa Elena, considerando que los parques temáticos son centros que brindan servicios de recreación lúdica cultural, y que por su gran dimensión y atracción son capaces de mover un amplio flujo de personas para ejecutar una actividad turística hacia una localidad, país o continente, es por esto que son considerados como productores de un gran impacto sobre los lugares donde se encuentran ubicados, debido a que se convierten en agentes estratégicos y de creación de lineamientos para un incremento tanto productivo como económico en el sector, a su vez, son generadores de puestos de trabajo con lo cual se crea una dinámica económica local y regional.

Estos parques temáticos se han convertido en instrumentos de desarrollo, porque promueven a que las instalaciones turísticas de una localidad crezcan y/o mejoren en sus servicios, debido a que la amplia demanda que se genera a costa de ellos, requiere que sus necesidades sean cubiertas con la respectiva oferta, sea esta tangible o intangible.

Pese a la existencia de algunos primeros parques cerrados y reservados únicamente a sus propietarios, el primer parque público con atracciones que se podría considerar el padre de los parques actuales es Bakken, situado en Copenhague, abierto en 1583 y que aún sigue en funcionamiento. En 1843, el parque de atracciones Tivoli Gardens, Copenhague, dio lugar a la creación de muchos parques homónimos por todo el mundo, constituidos como jardines con una gran cantidad de construcciones enfocadas al ocio. En la segunda mitad del siglo XIX, las nuevas tecnologías del hierro, el vapor y la electricidad pondrían de moda a finales de siglo los parques de atracciones basados en la velocidad y la fuerza centrífuga. La creciente riqueza de la sociedad europea y norteamericana de la época hace aparecer una civilización del ocio y con ella una era dorada para los parques de atracciones.

Aunque ya existían en Europa parques con atracciones mecánicas desde finales del siglo XVIII como el parque del Prater de Viena creado en 1766, los parques de atracciones concebidos como negocio de «diversión permanente» nacen a finales del XIX en Norteamérica. Concretamente el primer parque de atracciones moderno fue inaugurado en 1887 en Conney Island (Nueva York), y muy pronto en la periferia de las ciudades norteamericanas las compañías de tranvías y trolebuses abrieron numerosos parques de estas características en la proximidad de los extremos de las líneas cubiertas por esos modernos medios de transporte urbano eléctrico que, lógicamente se ubicaban en las periferias urbanas, como forma de crear centros de atracción de tráfico hacia esas zonas poco habitadas y rentabilizar las líneas.

\subsection{Referente teórico:}

"Entendidos como ciudades de ocio en las que se concentra un amplio concepto lúdico-cultural en el que los visitantes pueden pasar un día completo de diversión. Además, la Asociación Internacional de Parques de Diversiones y Atracciones (IAAPA) citado por (Rivera, 2015), menciona que un parque temático es "Un parque recreativo que tiene una o varias identidades temáticas que determinan las distintas alternativas en materia de atracciones, restauración, comercio, entre otras."

Con lo expuesto por expertos en el tema se puede determinar que los parques temáticos son lugares con gran dimensión en cuya área se exhibe una temática o temáticas específicas atrayentes e innovadoras que se exponen a través de un conjunto de atracciones con fines de ocio, entretenimiento y educación cultural, brindando a su vez los servicios complementarios como alimentación, comercio, entre otros al visitante, para que de esta manera pueda pasar un día placentero en dicho lugar.

A su vez los parques temáticos son centros de esparcimiento que aseguran al visitante una larga duración de su estancia donde sea capaz de generar gozo y aprendizaje a través de cada actividad efectuada por las personas que los visitan, pues a través del aspecto lúdico escenifican temas de importancia mundial creando así conciencia sobre ellos. 
Davis (1997) observa una variedad de énfasis, algunos contradictorios, en el análisis académico de los parques temáticos a mediados de los años setenta en los Estados Unidos. Inicialmente, los estudios académicos, centrados en gran medida en Disney, criticaban los parques temáticos como un resultado característico de la política económica norteamericana. Schiller (1973) citado por (Clave, 2007) fue influenciado intensamente por el radicalismo popular de finales de los años $60 \mathrm{y}$ fue probablemente el primer analista en criticar la supuesta postura de inocencia y neutralidad de los productos de entretenimiento de Disney. Sin embargo, este enfoque es abandonado y, a partir de los años ochenta se divide en dos corrientes. Por un lado, los historiadores sociales comienzan a abordar los parques temáticos desde el ángulo del análisis de los cambios en las tendencias de los consumidores durante el tiempo libre de la sociedad y se interesan, desde este punto de vista, en la historia de los parques temáticos.

Por otro lado, la interpretación de los parques temáticos como escenarios narrativos y textuales se generaliza. De esta manera, los antropólogos y semiólogos consideran los parques temáticos como espacios supuestamente paradigmáticos de la era posmoderna (Clave, 2007).

El turismo como se conoce es un fenómeno dinámico social que abarca un conjunto de relaciones humanas, debido a que produce un mover de personas de un lugar a otro, con la necesidad de satisfacción de un servicio por motivos placenteros y/o profesionales que involucran recreación, restauración, alojamiento, transporte $\mathrm{u}$ otros que directa $\mathrm{o}$ indirectamente se encuentran envueltos en la actividad turística, la cual funciona como un sistema integrador.

La aceleración del consumo y su preeminencia como motor económico a partir de los años ochenta, junto con la manifestación de los aspectos más crudos de la crisis estructural que afrontan las ciudades norteamericanas, despierta a los arquitectos, urbanistas, geógrafos y sociólogos A partir de los años noventa - en los parques temáticos desde la perspectiva de las relaciones entre la vida social y la producción del espacio. Desde este punto de vista, los parques temáticos son más que espacios de ocio. Se convierten en la expresión física de los deseos de la sociedad en cuanto a sus preferencias por la ciudad y ofrecen alternativas a los procesos convencionales de construcción urbana, que avanza hacia la privatización empresarial de los espacios y, en definitiva, la redefinición del espacio social. Sin embargo, este enfoque ha conducido a resultados diametralmente opuestos.

Así, mientras que Rebori (1995) mencionado por (Clave, 2007) sostiene que el estudio de Disney presenta numerosas ideas y ejemplos de aplicaciones en los campos de planificación, diseño, desarrollo y gestión de flujos, autores como Sorkin (1992a) usan esta misma base para criticar, Por medio de un recorrido por los principales escenarios de la geografía urbana contemporánea en América del Norte (las ciudades "analógicas" de Atlanta, Calgary y Montreal, el área comercial de West Edmonton Mall, la metrópolis de Los Ángeles o la utopía de Disneylandia) del espacio público o, al menos, la confusión entre el público y el privado.

De hecho, no se debe pasar por alto el hecho de que la parte más relevante de la bibliografía existente hasta la fecha en los parques temáticos se ha concentrado principalmente en Disney. En cualquier caso, el dominio de Disney de la industria del parque temático trae consigo dificultades adicionales en el estudio de los parques temáticos. Como símbolo corporativo del proceso de la "americanización del mundo", instigador de la expansión de la sociedad de consumo y rito de iniciación del capitalismo totalitario (Clave, 2007) menciona en su libro que (Aries, 2002, 8-9), asevera que Disney ha generado una multitud de interpretaciones críticas y que los más significativos de Klugman, Kuenz, Waldrep y Willis en 1995 bajo el nombre colectivo de The Project on Disney, 1995), han llevado a un deterioro de la capacidad de interpretar el fenómeno del parque temático más allá de Disney.

Así, para citar uno de los más recientes argumentos enérgicos contra la presencia de Disney en Francia, Aries (2002, 24) mencionado por (Clave, 2007) justifica tal preeminencia cuando dijo que "se ha convertido junto con McDonald's, IBM, Microsoft y Monsanto en uno de los Símbolos más importantes de la globalización capitalista".

Los primeros parques de atracciones americanos tenían un marcado carácter local, y poco después de éstos aparecerían otros que buscarían reunir a una gran cantidad de público dispuesto a enfrentarse a innovaciones de todo tipo. El moderno concepto de parque temático procede de la evolución que han experimentado los parques de recreo y las diversiones colectivas en un proceso de creciente integración que pasa por el eje central de los parques de atracciones que son su antecedente más inmediato.

Los parques temáticos se han popularizado en todo el mundo, tanto en países industrializados como en vías de desarrollo, porque atraen a una gran población, especialmente infantil $y$ juvenil $y$ son una oportunidad para crear conciencia acerca de temas que antes fueron relegados al espacio de la escuela, como la ciencia y las matemáticas, temas de preocupación mundial, como la ecología o temas vistos como restringidos a una clase intelectual, como la tecnología, la antropología, la geología y otros. Los 
parques temáticos son denominados como el conjunto de atracciones y espacios de ocio, entretenimiento, educación, cultura, entre otros, normalmente organizados y enfocados a un perfil que sirve de inspiración sobre la temática.

Las características de los parques temáticos según estudios realizados, entre ellos el de Economics Research Associates, determinan y ponen a manifiesto las siguientes características comunes que comparten estos centros de recreación:

Como primera característica se menciona que los parques temáticos son atractivos familiares. La oferta se adapta para los diferentes grupos de edad, convirtiéndolos en multi-atractivos según la demanda, dicha peculiaridad hace posible que se conserven abiertos todo el año.

En segundo lugar, presentan entre uno o más ambientes temáticos. Por lo general, estos contenidos varían según el tamaño del parque, su configuración y objetivo para el que ha sido creado. Como tercera característica se menciona la presencia de instalaciones de entretenimientos, es decir, atracciones y espectáculos públicos, actores, músicos ambulantes, disfraces, entre otros. En cuarto lugar, lo que caracteriza a los parques temáticos se encuentra representado por una fuerte inversión, dicho capital variará dependiendo de la capacidad de acogida para la cual son visionados. Otra característica manifestada es una política de precio único, donde el visitante solo pagará el costo de la entrada y podrá tener acceso a todos los atractivos que en él se encuentre.

Podemos entonces inferir, que los parques temáticos son centros de esparcimientos con temáticas específicas que generan ambientes de entretenimiento tanto para niños, jóvenes, y adultos contribuyendo así al disfrute familiar, considerando además que las existencias de los parques influyen a la creación de comerciales alrededor de los mismos.

Los Parques Temáticos en el Ecuador se han venido desarrollando en gran parte dentro de entidades públicas y privadas, claros ejemplos que se pueden tomar como referencia de los parques en el Ecuador, son: El "Vulcano Park" que se encuentra ubicado en la ciudad de Quito, provincia de Pichincha, el Parque Histórico de Guayaquil ubicado en la provincia del Guayas, y el Parque de los Picapiedras ubicado en la ciudad de Machala, provincia El Oro, son parques con temáticas diferentes pero que aportan para que el lugar sea atractivo al turista.

Los parques temáticos o el turismo temático es un potencial destino turístico muy visitado y atraído por una gran población inclinada a la actividad del turismo donde su principal característica es atraer turistas mediante tematización, diseño, desarrollo y factores de éxito que se deben considerar al momento de planificarlos además del cumplimiento de los objetivos planteados en el mismo y sobre todo se beneficie y aporten al desarrollo local.

\section{Materiales y Métodos}

La investigación es cuali-cuantitativa pretendiendo generalizar el estudio de campo orientado a la solución práctica, factible y eficaz del objeto, siendo necesaria la definición metodológica fundamentada en el método Histórico Inductivo - Deductivo, mediante el conocimiento de las etapas de los objetos en su sucesión cronológica, permitiendo así conocer la evolución y desarrollo del problema, es decir, la observación de trayectoria concreta y la esencia histórica, mediante la investigación acción participativa.

La recolección de datos primarios se efectuó mediante la aplicación de instrumentos como la encuesta a pobladores de la ciudad de Santa Elena y turistas y servidores turísticos de la Provincia del mismo nombre, además de entrevistas realizadas a autoridades del cantón, tomados en consideración ya que son parte fundamental como beneficiarios del trabajo de estudio además es de suma importancia saber las opiniones vertidas por ellos acerca del mismo.

\section{Población y muestra}

El universo poblacional que sirvió como unidad de análisis estuvo conformado por:

\begin{tabular}{|c|c|c|}
\hline SEGMENTO & $\begin{array}{l}\text { CANTIDAD } \\
\text { UNIVERSO }\end{array}$ & FUENTE \\
\hline Turistas & 33.616 & REMACOPSE \\
\hline $\begin{array}{l}\text { Población de la } \\
\text { ciudad de Santa } \\
\text { Elena }\end{array}$ & 39.681 & $\begin{array}{l}\text { Plan de Desarrollo y } \\
\text { Ordenamiento Territorial } \\
2012 \text {-2019 (Santa Elena) }\end{array}$ \\
\hline $\begin{array}{l}\text { Servidores } \\
\text { turísticos }\end{array}$ & 6 & $\begin{array}{l}\text { Dirección Provincial de } \\
\text { Turismo de Santa Elena }\end{array}$ \\
\hline $\begin{array}{l}\text { Autoridades } \\
\text { locales }\end{array}$ & 2 & $\begin{array}{l}\text { GAD Municipal de Santa } \\
\text { Elena (Alcaldía) }\end{array}$ \\
\hline TOTAL & 73305 & \\
\hline
\end{tabular}

Se consideró adecuado aplicar el muestreo probabilístico aleatorio simple, con el fin de que las personas involucradas tengan la misma probabilidad de ser seleccionadas.

Aplicación de la fórmula:

$$
n=\frac{Z^{2} p q * N}{e^{2}(N-1)+Z^{2} p q}
$$

Donde: 


\begin{tabular}{|l|r|r|c|}
\hline \multicolumn{1}{|c|}{ SEGMENTO } & $\begin{array}{r}\text { CANTIDAD } \\
\text { UNIVERSO }\end{array}$ & $\begin{array}{c}\text { TAMAÑO } \\
\text { MUESTRAL }\end{array}$ & INSTRUMENTO \\
\hline Turistas & 33616 & 138 & Encuesta \\
\hline $\begin{array}{l}\text { Población de la } \\
\text { ciudad de Santa } \\
\text { Elena }\end{array}$ & 39681 & 138 & Encuesta \\
\hline Servidores turísticos & 6 & 6 & Encuesta \\
\hline Autoridades locales & 2 & 2 & Entrevista \\
\hline \multicolumn{1}{|c|}{ Total } & $\mathbf{7 3 3 0 5}$ & $\mathbf{2 7 7}$ & Encuesta/Entrevista \\
\hline
\end{tabular}

\section{Procedimiento y procesamiento de la Investigación}

El procedimiento y procesamiento que se desarrollaron en la presente investigación fueron los siguientes:

- Búsqueda de la Información

- Marco contextual

- Formulación de la metodología

- Elaboración del instrumento de Investigación Gestión y apoyo institucional,

En lo que corresponde al procesamiento se realizó:

- Ordenamiento de los datos

- Codificación de datos

- Tabulación de datos

- Elaboración de tablas y gráficos estadísticos

- Análisis e interpretación de resultados

La información recopilada se basó en:

Encuestas a Turistas. - Compuesta de un total de 19 ítems o preguntas, su objetivo fue obtener información concerniente a datos básicos personales del visitante, información turística que sirvió para determinar cualicuantitativamente el grado de interés que los visitantes tienen acerca del turismo Cultural e Histórico, además de recabar información relevante que permita determinar nuevas alternativas de diversificación de la oferta turística.

Encuestas a Pobladores. - Compuesta de un total de 14 ítems o preguntas, su objetivo fue obtener información concerniente a datos básicos personales del poblador, información turística para determinar cualicuantitativamente el grado de aceptación e involucramiento social que los habitantes de la ciudad Santa Elena tienen con los Proyectos Turísticos Cultural e Históricos, además de recabar información relevante sobre la demanda turística local.
Encuestas a Servidores Turísticos Compuesta de un total de 18 ítems o preguntas, su objetivo fue obtener información concerniente a datos básicos personales del servidor turístico, información turística que sirvió para determinar cuali-cuantitativamente el grado de involucramiento que los servidores turísticos tendrían con la Creación de un Parque Temático Recreativo Turístico - Cultural, en la ciudad de Santa Elena, además se logró obtener información relevante sobre la demanda turística del lugar.

Entrevista a las Autoridades Locales Compuesta de un total de 7 ítems o preguntas, su objetivo fue determinar la opinión y compromiso de las Autoridades, al ser consideradas como directos responsables del desarrollo turístico nacional y local en la aplicación de la Creación de un Parque Temático Recreativo Turístico - Cultural, en la ciudad de Santa Elena.

\section{Resultados}

Patrimonio cultural Inmaterial de la Ciudad de Santa Elena como potencial Turístico. Las autoridades entrevistadas están de acuerdo con que la ciudad de Santa Elena es potencial turístico en historia y cultura, ya que posee suficientes antecedentes que se podrían fomentar a través de una oferta turística compatible a estos recursos como lo es el parque temático.

Las sugerencias para la planificación de la creación de un Parque Temático Recreativo Turístico fueron orientadas a la realización de un diagnóstico claro para de esta manera, identificar las potencialidades Culturales, y establecer claramente cuál sería la demanda del producto, es decir a que público estaría dirigido.

Respecto al apoyo del proyecto de la creación del parque temático los entrevistados respondieron unánimemente que ellos apoyarían especialmente en la Promoción del Parque Temático, además, en la coordinación y en la aplicación de un modelo de gestión.

La Empresa Municipal de Turismo del cantón Santa Elena (EMUTURISMO) y a la Dirección de Turismo de Santa Elena, de las respuestas obtenidas por parte de sus representantes en la provincia y cantón Santa Elena se puede determinar un gran apoyo y aceptación de la Creación del Parque Temático Histórico - Cultural que se propone, al considerar y estar conscientes de que Santa Elena tiene un gran potencial, pero sobre todo que es cuna histórica de la península, por lo cual hay mucho que ofrecer, muchas alternativas que se pueden generar alrededor del tema Histórico - Cultural, con la finalidad de dar valor a los mismos, pero partiendo del mejoramiento de los servicios turísticos y básicos de la ciudad, y en base a esto brindar o aportar con un mejor estilo de vida a la comunidad, pues se pretende un 
desarrollo no solo turístico sino también socioeconómico del sector.

En cuanto a las encuestas realizadas a la población y a los turistas si visitarían a un parque temático en la ciudad de Santa Elena, las respuestas fueron favorables ya que, ellos si visitarían una nueva y atractiva oferta turística, ya que, a más de ser un producto llamativo para la población de Santa Elena, es un producto novedoso, que podría ser muy visitado por turistas nacionales y extranjeros enfocados a diferentes segmentos de mercado, es decir para todo tipo de turista. Por lo que, esta nueva y atractiva oferta turística beneficiaría a la población mediante la gran afluencia turística, mejoría la calidad de vida, ente fundamental para la fuente de trabajo, y entre otras alternativas que aportan al desarrollo de la localidad.

\section{Conclusiones}

En la ciudad de Santa Elena, a pesar de la existencia del museo "Amantes de Sumpa" con enfoque cultural no existe un lugar o atractivo turístico con temática que conjugue lo recreativo y cultural, representando costumbres y tradiciones locales para el disfrute de los visitantes. Existen recursos con grandes potencialidades a nivel cultural en la provincia de Santa Elena, sin embargo, están quedando rezagadas porque los habitantes no se sienten identificados con su propia cultura, pero que se pueden utilizar para satisfacer las tendencias que exige el turista actual con buena planificación y gestión turística.

\section{Bibliografía}

Asociación Internacional de Parques de Diversiones y Atracciones (IAAPA) citado por Fuertes, A. M., \& Molina, C. A. (2000). Los Parques Tematicos como estrategia de producto. Recuperado el 14 de Julio de 2014, de Cuadernos de ciencias Económicas y Empresariales, ISSN 0211-4356, N³9, 2000:

http://dialnet.unirioja.es/servlet/articulo?codi go $=208243$

Clave, S. A. (2007). THE GLOBAL THEME PARK INDUSTRY. USA, Massachusetts: CABI North American.

Davis, G. S. (1997). Spectacular Nature. Berkeley, California: University of California Press.

Fundación Santiago de Guayaquil. (2011). PLAN DE DESARROLLO Y ORDENAMIENTO TERRITORIAL 2012-2019. Santa Elena.
Gomes, C. L., \& Rodrigo, E. (2012). Horizontes latino-americanos do lazer / Horizontes latinoamericanos del ocio. Belo Horizonte: UFMG.

Instituto Nacional de Estadísticas y Censos, INEC. (2010). Censo 2010 Santa Elena.

Londoño, G. (23 de Julio de 2009).

http://www.tesisenred.net. Obtenido de http://www.tesisenred.net:

http://www.tesisenred.net/bitstream/handle/1 0803/9646/londono.pdf?sequence $=1$

MINTUR - Ley No.97. Actualizada . (06 de Mayo de 2008). LEY DE TURISMO. Recuperado el 10 de 08 de 2014, de LEY DE TURISMO: http://www.turismo.gob.ec/wpcontent/uploads/downloads/2014/02/Ley-deTurismo-MINTUR.pdf

MINTUR y T \& L. (26 de Septiembre de 2007). PLANDETUR 2020. Recuperado el 10 de Agosto de 2014, de PLANDETUR 2020: http://www.turismo.gob.ec/wpcontent/uploads/downloads/2013/02/PLAND ETUR-2020.pdf

Organización Mundial de Turismo, citado en Secretaría de Turismo, Programa Nacional de Turismo 2001-2006. (2001). T. México.

Pearce, D. (1998). Desarrollo Turístico: su planificación y ubicación geográficas. México: Trillas S.A.

Ritzer citado por Clavé, S. A. (1999). El desarrollo de parques temáticos en un contexto de globalización. Boletín de la Asociación de Geógrafos Españoles, 85-102. Recuperado el 14 de Julio de 2014, de dialnet.unirioja.es/descarga/articulo/1318650. pdf

Rivera, L. y. (8 de Diciembre de 2015). Creación de un parque temático recreativo turístico cultural, cantón Santa Elena, provincia Santa Elena, año 2015 . La Libertad, Santa Elena, Ecuador.

Sanchez, J. (10 de Junio de 2013). ANÁLISIS COMPARATIVO DE LA ACTIVIDAD TURÍSTICA. Malaga, Malaga, España. 
Senplades. (24 de Junio de 2013). buenvivirPLAN

NACIONAL2013 - 2017. todo el mundo mejor. Recuperado el 10 de 08 de 2014, de buenvivirPLAN NACIONAL2013 - 2017. todo el mundo mejor:

http://www.buenvivir.gob.ec/presentacion

Ten, A. (1998). LOS NUEVOS PARAISOS. HISTORIA Y EVOLUCION DE LOS PARQUES TEMATICOS. Recuperado el 19 de julio de 2014, de http://www.uv.es/ten/p831.html
Trilla citado por Muñoz Corvalán, J. (julio de 2012). "El ocio y tiempo libre en la sociedad actual", en Cotribuciones a las Ciencias Sociales. Recuperado el 24 de junio de 2015, de sitio web de:

www.eumed.net/rev/cccss/20/ 